\title{
Redshift limits of BL Lacertae objects from optical spectroscopy (Research Note)
}

\author{
J. D. Finke ${ }^{\star, \star \star}$ J. C. Shields, M. Böttcher, and S. Basu
}

\author{
Astrophysical Institute, Department of Physics and Astronomy, Ohio University, Athens, Ohio, 45701, USA \\ e-mail: jfinke@ssd5.nrl.navy.mil
}

Received 16 August 2007 / Accepted 29 October 2007

ABSTRACT

\begin{abstract}
Context. BL Lacertae objects have been the targets for numerous recent multiwavelength campaigns, continuum spectral variability studies, and theoretical spectral and variability modeling. A meaningful interpretation of the results of such studies requires a reliable knowledge of the objects' redshifts; however, the redshifts for many are still unknown or uncertain.

Aims. Therefore, we hope to determine or constrain the redshifts of six BL Lac objects with unknown or poorly known redshifts. Methods. Observations were made of these objects with the MDM $2.4 \mathrm{~m}$ Hiltner telescope. Although no spectral features were detected, and thus no redshifts could be measured, lower redshift limits were assigned to the objects based on the expected equivalent widths of absorption features in their host galaxies. Redshifts were also estimated for some objects by assuming the host galaxies are standard candles and using host galaxy apparent magnitudes taken from the literature.

Results. The commonly used redshift of $z=0.102$ for $1219+285$ is almost certainly wrong, while the redshifts of the other objects
\end{abstract} studied remain undetermined.

Key words. galaxies: BL Lacertae objects: general - galaxies: distances and redshifts

\section{Introduction}

Blazars are among the most violent, highly variable astrophysical high-energy phenomena, with rapidly-varying emission from radio through $\gamma$-ray energies. They are thought to consist of relativistic jets from supermassive black holes, closely aligned with our line of sight.

BL Lacertae (BL Lac) objects, a subclass of blazars, are defined by their quasar-like continuum and the weakness or absence of broad emission lines in their optical spectra. These spectra are thought to be dominated by nonthermal emission from the highly relativistic jet, masking the contributions of the stars in the host galaxy, and line emission from gas clouds near the supermassive black hole.

There are various models for the source of the nonthermal emission from blazars. Of critical importance in distinguishing between the models is setting the energy scale, on which many parameters (e.g., the jet's speed, density, magnetic field, etc.) depend. This can only be done if the objects' redshifts, and hence distances, are known.

Many well-observed BL Lac objects have unknown or poorly known redshifts based on $\sim 1-3$ emission or absorption lines in low signal-to-noise $(S / N)$ spectra. In several cases, a poorly determined redshift of a BL Lac is repeatedly cited throughout the literature until its reliability is no longer questioned. Thus, a project was undertaken to obtain the optical spectra of several of these objects at the MDM Observatory with the goal of obtaining redshift estimates or improved constraints.

Unfortunately, no definitive spectral features were revealed, and thus no definitive redshifts could be assigned. However,

* Current Address: Code 7653, US Naval Research Laboratory, Washington, DC, 20375, USA.

$\star \star$ NRC/NRL Research Associate. using the method of Sbarufatti et al. (2006), we were able to estimate a minimum redshift based on the expected equivalent widths of absorption features in the host galaxy. Also, assuming the host galaxies are standard candles (see, e.g., Nilsson et al. 2003), we estimated the redshifts of several objects based on the observed magnitude of the host galaxies, found from the literature, based on the method of Sbarufatti et al. (2005).

\section{Observations and data analysis}

\subsection{Observations}

Spectroscopic observations of six BL Lac objects were taken with the MDM 2.4 m Hiltner telescope in November 2005 and March 2006. Arc lamps and one standard star per night per wavelength setting were also observed for wavelength and spectrophotometric calibration, respectively. The CCDS spectrograph was used with a slit width of $1.5^{\prime \prime}$ and the 350 grooves $/ \mathrm{mm}$ grating. The grating provides a wavelength range of $1592 \AA$. Three different observing settings were used: 4000-5500 (hereafter referred to as the blue setting), 5500-7000 (green setting) and $\sim 7000-8500 \AA$ (red setting). For the red and green settings, the LG-400 order-blocking filter was used. Unfortunately, weather conditions did not allow observation of all the sources with all of the settings. Seeing during the observations was in the range $\sim 1-3^{\prime \prime}$ with an average of $\sim 1.5^{\prime \prime}$. On 29 November conditions were nearly photometric; on the other nights, thin cirrus drifted in and out of the field. On 29 November the seeing was $\sim 2^{\prime \prime}$, and the slit was not consistently aligned with the parallactic angle, leading to noticeable flux losses from atmospheric differential refraction. A summary of the observations can be found in Table 1 . 
Table 1. Blazar observations with the Hiltner telescope.

\begin{tabular}{rrrccc}
\hline \hline Object & RA (J2000) & Dec (J2000) & Setting & Exp. time [s] & Obs. date (UT) \\
\hline $0219+428$ & $02: 22: 39.6$ & $+43: 02: 08$ & blue & 4800 & 29 Nov. 2005 \\
& & & green & 7200 & 29 Nov. 2005 \\
& & & blue & 3600 & 30 Nov. 2005 \\
& & red & 10800 & 30 Nov. 2005 \\
$0716+714$ & $07: 21: 53.4$ & $+71: 20: 36$ & green & 7200 & 29 Nov. 2005 \\
& & & blue & 5400 & 30 Nov. 2005 \\
$1011+496$ & $10: 15: 04.1$ & $+49: 26: 01$ & blue & 7200 & 26 Mar. 2006 \\
$1055+567$ & $10: 58: 37.7$ & $+56: 28: 11$ & blue & 7200 & 26 Mar. 2006 \\
$1219+285$ & $12: 21: 31.7$ & $+28: 13: 59$ & blue & 5400 & 26 Mar. 2006 \\
$1426+428$ & $14: 28: 32$ & $+42: 40: 21$ & blue & 5090 & 26 Mar. 2006 \\
\hline
\end{tabular}

Table 2. Results of blazar observations.

\begin{tabular}{rccccc}
\hline \hline Object & $m_{B}$ & $S / N$ & $E W_{\min }[\AA]$ & $z_{\text {spec }}$ & $z_{\text {phot }}$ \\
\hline $0219+428$ & $15.35 \pm 0.50$ & 90 & 0.87 & $\geq 0.096$ & 0.321 \\
$0716+714$ & $13.39 \pm 0.50$ & 270 & 0.27 & $\geq 0.070$ & - \\
$1011+496$ & $15.80 \pm 0.02$ & 50 & 1.001 & $\geq 0.134$ & $0.213 \pm 0.041$ \\
$1055+567$ & $15.60 \pm 0.02$ & 70 & 0.79 & $\geq 0.136$ & - \\
$1219+285$ & $14.88 \pm 0.50$ & 90 & 0.43 & $\geq 0.104$ & $0.161 \pm 0.035$ \\
$1426+428$ & $17.10 \pm 0.50$ & 10 & 3.17 & $\geq 0.106$ & $0.132 \pm 0.030$ \\
\hline
\end{tabular}

The spectra were reduced with IRAF $^{1}$ using standard methods. For each object and grating setting, 3-4 spectra were taken, which were then averaged into a single spectrum.

\subsection{Redshift limit procedure}

The procedure for finding the minimum redshift of the BL Lac objects is described by Sbarufatti et al. (2006) We refer the reader to that work, and point out that their Eq. (1) contains a typographical error (Finke 2007) and should be

$$
E W_{\mathrm{obs}}=\frac{(1+z) E W_{0}}{1+\rho(\lambda) / A(z)}
$$

where $E W_{0}$ is the equivalent width of a line in the rest frame of the galaxy, $\rho$ is the AGN flux to host galaxy flux ratio and $A(z)$ is the aperture correction, which takes into account the fact that not all of the galaxy's emission will be in the extraction aperture.

To determine the lower limit, one needs two observations: the minimum possible $E W$ one could observe with the spectrum, and the appearent magnitude of the object (both these things are listed in Table 2). For the objects $1011+496$ and $1055+567$, photometric observations on the same night were taken with the McGraw-Hill $1.3 \mathrm{~m}$ telescope. For the rest of the spectra, $m_{B}$ was determined by integrating the spectrum weighted by the response function for the $B$ filter using the IRAF utility SBANDS. The magnitudes of $1011+496$ and $1055+567$ taken from photometric observations were compared with the magnitudes derived from the spectra and found to vary by less than 0.5 mag. We compared the standard star observation on 26 March with an exposure of that star aborted due to losing the guide star by heavy cloud cover. The difference was $\sim 0.52 \mathrm{mag}$. We consequently adopted $0.5 \mathrm{mag}$ as a reasonable estimate of the uncertainty in spectroscopic magnitudes for the other targets.

${ }^{1}$ IRAF (Image Reduction and Analysis Facility) is distributed by the National Optical Astronomy Observatory which is operated by the Association of Universities for Research in Astronomy, Inc., under cooperative agreement with the National Science Foundation.

\section{Results}

Flux-calibrated and normalized spectra for our sources are seen in Figs. 1 and 2 and results are sumarized in Table 2. In a few instances the spectra show residual structure that we attribute to imperfect flux calibration (e.g., at $\sim 4880 \AA$ in the 2005 spectra and $\sim 4500 \AA$ in the 2006 spectra), and variations of slope between grating settings of similar origin. Low-frequency variations of this type do not significantly impact our analysis.

A brief description of the previous observations and results for individual objects are discussed below. All error bars quoted are $1 \sigma$ error bars.

$0219+428(3 \mathrm{C} \mathrm{66A)}$ ) has been the target of a recent multiwavelength campaign (Böttcher et al. 2005) and has been extensively observed in the radio to $\gamma$-rays. Its optical spectrum was first observed by Wills \& Wills (1974), who found it to be flat and featureless. Observations by Miller et al. (1978) with the Lick 3 m Shane reflector revealed an emission feature at $4044 \AA$, which they identified as $\mathrm{Mg}$ II 2800, giving the object a redshift of $z=0.444$. However, the feature is located in a region where it is confused with telluric $\mathrm{H}_{2} \mathrm{O}$ absorption, and the authors did not consider it reliable. International Ultraviolet Explorer observations detected a feature at $1750 \AA$ which could be Ly $\alpha$ emission at a redshift of $z=0.444$, but the redshift of $0219+428$ is still far from certain (Lanzetta et al. 1993). Its host galaxy was marginally resolved by Wurtz et al. (1996), and found to have a magnitude of $r_{\mathrm{Gunn}}=19.0$. Converting to Johnson $R$ using the prescription of Kent (1985) yields $m_{R}=18.43$, and thus a photometric redshift of $z=0.321$. Wurtz et al. (1996) do not provide an error estimate for the magnitude; however, since it was only marginally resolved, the error can be assumed to be high. Our spectrum's wavelength range does not cover the range of the Miller et al. (1978) feature, so we are unable to confirm or refute their detection; however, we were able to constrain $0219+428$ 's redshift to $z \geq 0.096$. The $0219+428$ green spectrum was likely affected by slit losses due to atmospheric dispersion, which would explain the difference in slope from the red and blue spectra. Although we removed most telluric features, 

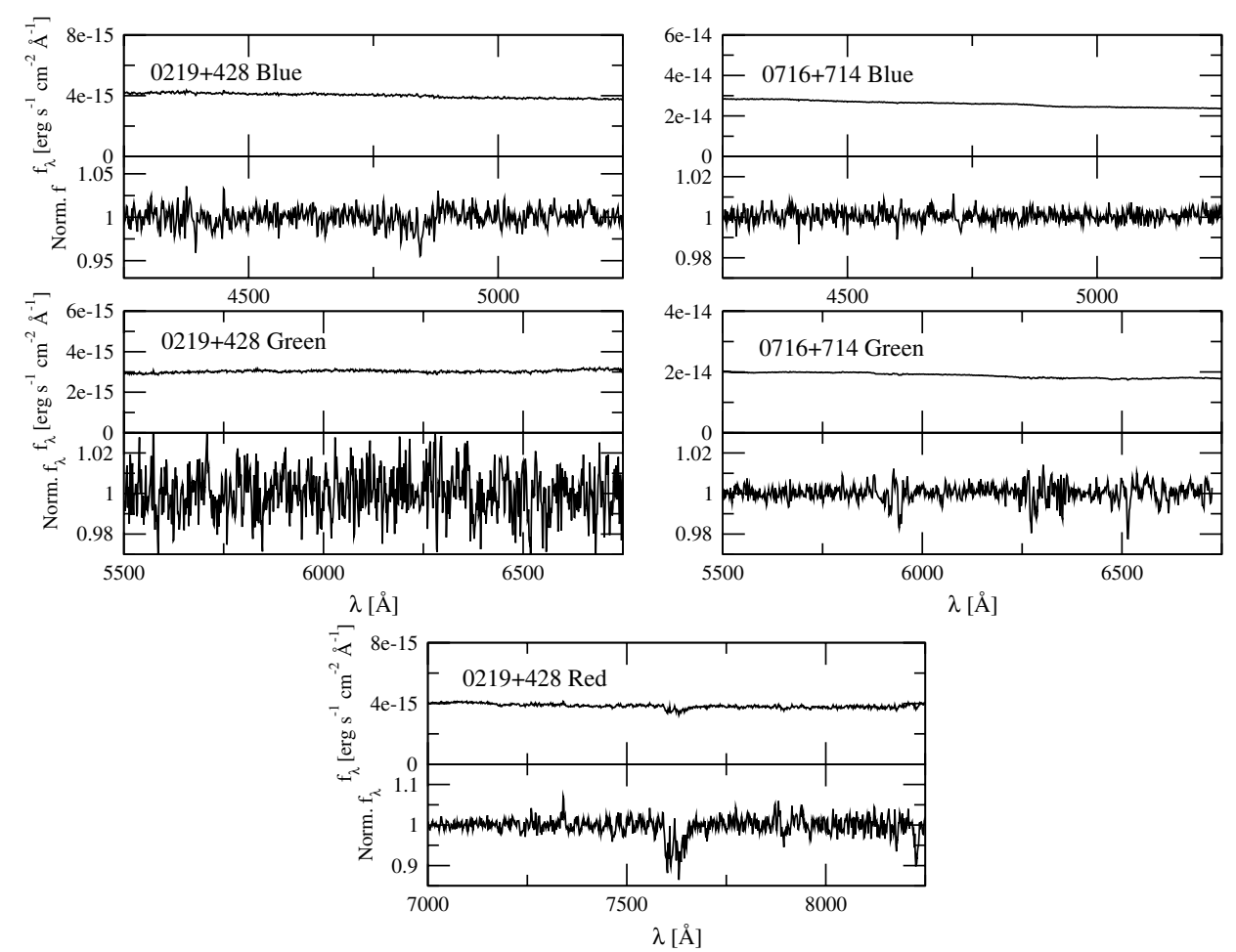

Fig. 1. Blazar spectra taken in 2005.
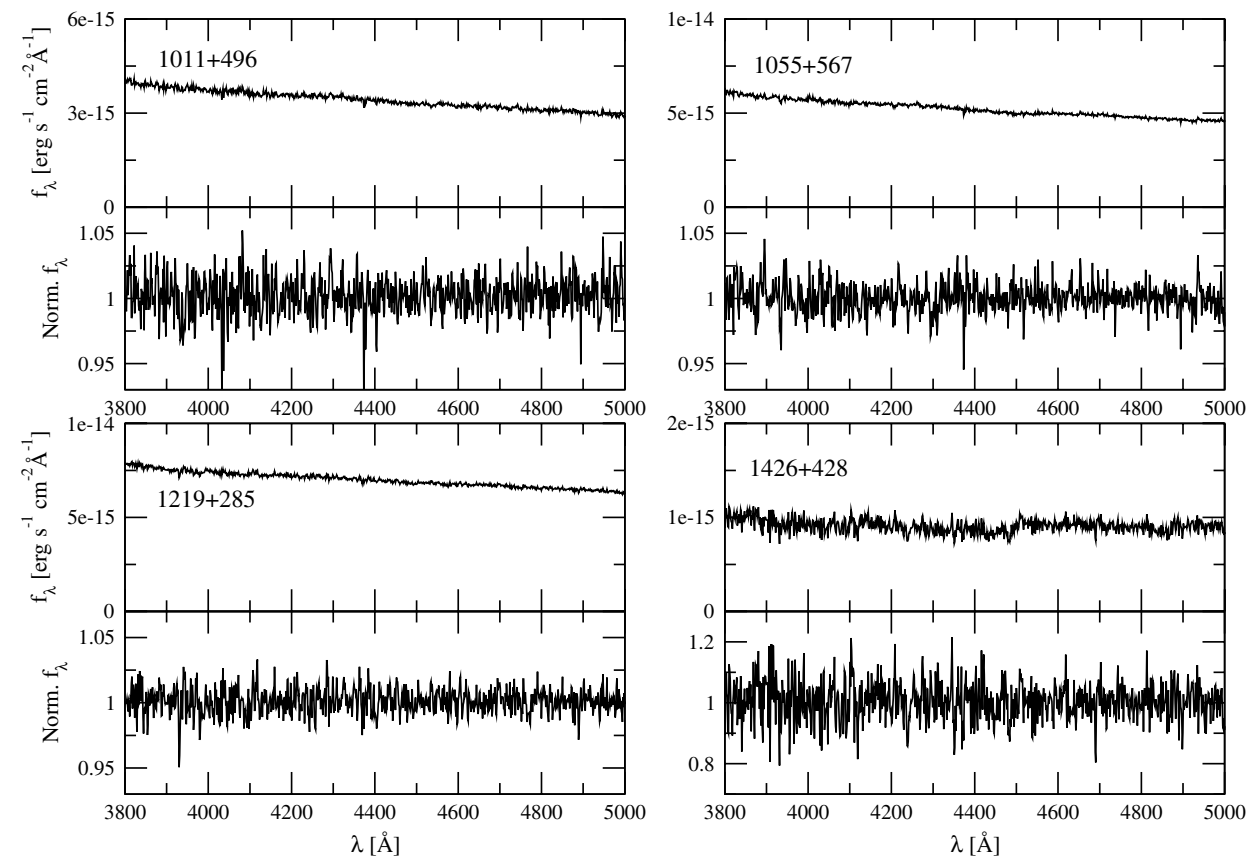

Fig. 2. Blazar spectra taken in 2006.

we were unable to fully remove the A band feature (7594 $\AA$ ) in the red spectrum.

PKS 0716+714 has been detected in X-rays and $\gamma$-rays (see, e.g., Foschini et al. 2006). High $S / N$ spectra taken with the KPNO $2.1 \mathrm{~m}$ and MMT $6.5 \mathrm{~m}$ telescopes are flat and featureless (Rector \& Stocke 2001). Our observations also did not reveal any spectral features, and we constrained its redshift to $z \geq 0.070$; despite its high $S / N$, its brightness does not allow for a higher constraint. Its host galaxy is unresolved, and Sbarufatti et al. (2005) used this to constrain its redshift to $z \geq 0.52$.
1011+496 has been detected by EGRET (Thompson et al. 1995), BeppoSAX (Donato et al. 2005) and MAGIC (Albert et al. 2007). Its last published spectrum was obtained with the McDonald Observatory $2.7 \mathrm{~m}$ telescope (Machalski 1991). Their spectrum was from $3200 \AA$ to $6000 \AA$ and included an unidentified feature at $\sim 3700 \AA$. Another spectrum with the KPNO $2.1 \mathrm{~m}$ telescope from 4400-6500 A showed no emission or absorption lines. Neither spectrum had a $S / N>5$. Its redshift is usually quoted as $z=0.20$, as this is the redshift of the nearby cluster, A950, to which $1011+498$ is presumed to belong 
(Leir \& van den Bergh 1977). With our higher $S / N$ spectrum we were also unable to detect any spectral features; however, we could constrain its redshift to $z \geq 0.134$. With a resolved host galaxy magnitude of $m_{R}=17.30$ from HST observations (Urry et al. 2000), its photometric redshift can be estimated to be $z=0.213 \pm 0.041$, in agreement with it being part of the cluster A950. 1011+496 has never had an optical spectrum published beyond $6500 \AA$.

$\mathbf{1 0 5 5 + 5 6 7}$ has also been detected by EGRET (Thompson et al. 1995) and BeppoSAX (Donato et al. 2005). Marchã et al. (1996) report a redshift of $z=0.410$ based on probable detection of the [O III] doublet in MMT spectra. A measurement by Bade et al. (1998) using the WHT, obtained with the source at a comparable flux level, does not appear to confirm the [O III] emission and the authors instead estimate $z=0.144$ based on Na I D absorption and blended $\mathrm{H} \alpha+[\mathrm{N}$ II $]$ emission. However, both features are very weak, and the putative emission feature is additionally suspect since it sits on the wing of the atmospheric A-band absorption feature. Our spectrum does not cover the wavelength range of these features, and we can only constrain the redshift to be $z \geq 0.136$. This object has not had an observation of its host galaxy published, thus we cannot estimate a photometric redshift. It should be noted that, although the $z=0.144$ value is cited more often in the literature (and is quoted as such in the Simbad database), the $z=0.410$ redshift is still used as well.

1219+285 (W Comae) has been detected by EGRET (Sreekumar et al. 1996) and is considered a promising target for Very High Energy (VHE) $\gamma$-rays by instruments such as VERITAS or MAGIC. Weistrop et al. (1985) performed spectroscopy on the object with the $4 \mathrm{~m} \mathrm{KPNO}$ telescope and estimated a redshift of $z=0.102$ based on [O III] and $\mathrm{H} \alpha$. However, the spectrum shows strong residuals due to sky subtraction and possibly other problems, and the authors acknowledge the line identifications are uncertain. Our high $S / N$ spectrum did not reveal any spectral features, but we did not observe in a range that would allow us to confirm the features detected by Weistrop et al. (1985). Nesci et al. (2001) published a spectrum of the object with the $2.6 \mathrm{~m}$ Byurakan Observatory telescope but were unable to confirm the observation of [O III] detected by Weistrop et al. (1985). The host galaxy of $1219+285$ was resolved by Nilsson et al. (2003), and they found its magnitude to be $m_{R}=$ $16.60 \pm 0.10$. Based on this measurement, we estimate its photometric redshift to be $z=0.161 \pm 0.035$, a considerable discrepancy with the spectroscopic value of Weistrop et al. (1985). We could spectroscopically constrain the redshift of $1219+285$ to $z \geq 0.104$. It therefore seems unlikely that the Weistrop et al. (1985) redshift of $z=0.102$ is correct.

$\mathbf{1 4 2 6}+\mathbf{4 2 8}$ has been detected at VHE $\gamma$-rays by CAT and HEGRA (Djannati-Ataï et al. 2002; Petry et al. 2000) and has been the target of multiwavelength campaigns (Horns 2003).
Its only reported optical spectrum was published by Remillard et al. (1989). Their highest $S / N$ spectrum $(S / N \sim 10)$ with the MDM $1.3 \mathrm{~m}$ telescope yielded $z=0.129$ from marginal detections of $\mathrm{Mg}$ I and $\mathrm{Na}$ I at $\sim 5800$ and $\sim 6650 \AA$. Unfortunately, we were not able to achieve a higher $S / N$ spectrum, nor were we able to observe at a wavelength above $5500 \AA$, thus we could not confirm this observation; we could only constrain its redshift to $z \geq 0.106$. The host galaxy of $1426+428$ was resolved by Urry et al. (2000) and found to have $m_{R}=16.14$, leading to a photometric redshift of $z=0.132 \pm 0.030$. Our measurements are consistent with the previous redshift claims.

\section{Summary}

The spectra of six BL Lac objects with poorly known or unknown redshifts have been obtained. For several objects, these spectra have higher $S / N$ than any previously published. Based on this papers' results, the commonly used redshift of $z=0.102$ for $1219+285$ is almost certainly wrong. The redshifts of the other objects studied remain undetermined.

Acknowledgements. We thank the anonymous referee for helpful comments and Jules Halpern for pointing out an error in Fig. 2.

\section{References}

Albert, J. 2007, ApJ, 667, L21

Bade, N., Beckman, V., Douglas, N. G., et al. 1998, A\&A, 334, 459

Böttcher, M., Harvey, J., Joshi, M., et al. 2005, ApJ, 631, 169

Donato, D., Sambruna, R. M., \& Gliozzi, M. 2005, A\&A, 433, 1163

Djannati-Ataï, A., Khelifi, B., Vorobiov, S., et al. 2002, A\&A, 391, L25

Finke, J. D. 2007, Ph.D. Thesis, Ohio University

Foschini, L., Tagliaferri, G., Pian, E., et al. 2006, A\&A, 455, 871

Horns, D. 2003, ASPC, 299, 13H

Kent, S. M. 1985, PASP, 97, 165

Lanzetta, K. M., Turnshek, D. A., \& Sandoval, J. 1993, ApJ, 84, 109

Leir, A. A., \& van den Bergh, S. 1977, ApJS, 34, 381

Machalski, M. 1991, AcA, 41, 39

Marchã, M. J., Brown, I. W. A., Impey, C. D., \& Smith, P. S. 1996, MNRAS, 281,425

Miller, J. S., French, H. B., \& Hawley 1978, in Pittsburgh Conference on BL Lac Objects, ed. A. M. Wolfe (Pittsburgh: Univ. Pittsburgh), 176

Nesci, R., Massaro, E., Rossi, C., et al. 2005, AJ, 130, 1466

Nesci, R., Massaro, E., Movsessian, T., \& Ohanian, G. 2001, Mem. Soc. Astron. It., 72, 145

Nilsson, K., Pursimo, T., Heidt, J., et al. 2003, A\&A, 400, 95

Petry, D., Böttcher, M., Connaughton, V., et al. 2000, ApJ, 536, 742

Rector, T. A., \& Stocke, J. T. 2001, ApJ, 122, 565

Remillard, R. A., Tuohy, I. R., Brissenden, J. V., et al. 1989, ApJ, 345, 140

Sbarufatti, B., Treves, A., \& Falomo, R. 2005, ApJ, 635, 173

Sbarufatti, B., Treves, A., Falomo, R., et al. 2006, ApJ, 132, 1

Sreekumar, P., Bertsch, D. L., Dingus, B. L., et al. 1996, ApJ, 464, 628

Thompson, D. J., Bertsch, D. L., Dingus, B. L., et al. 1995, ApJS, 101, 259

Urry, C. M., Scarpa, R., O’Dowd, M., et al. 2000, ApJ, 532, 816

Wills, B. J., \& Wills, D. 1974, ApJ, 190, L97

Weistrop, D., Hintzin, P., Shaffer, D. B., \& Romanishin, W. 1985, ApJ, 292, 614

Wurtz, R., Stocke, J. T., \& Yee, H. K. C. 1996, ApJS, 103, 109 